\title{
Shape Matching by Integral Invariants on Eccentricity Transformed Images
}

\author{
Faraz Janan, Sir Michael Brady
}

\begin{abstract}
Matching occluded and noisy shapes is a frequently encountered problem in vision and medical image analysis and more generally in computer vision. To keep track of changes inside breast, it is important for a computer aided diagnosis system (CAD) to establish correspondences between regions of interest. Shape transformations, computed both with integral invariants and geodesic distance yield signatures that are invariant to isometric deformations, such as bending and articulations. Integral invariants are used on 2D planar shapes to describe the shape boundary. However, they provide no information about where a particular feature on the boundary lies with regard to overall shape structure. On the other hand, eccentricity transforms can be used to match shapes by signatures of geodesic distance histograms based on information from inside the shape; but they ignore the boundary information. We describe a method that combines both the boundary signature of shape obtained from integral invariants and structural information from the eccentricity transform to yield improved results.
\end{abstract}

\section{INTRODUCTION}

Mathematically, a shape is considered to be a closed contour that describes a single entity. Shape matching is a wellknown problem in the field of medical image analysis and computer vision. It is usually performed by producing a shape signature, which ideally is invariant to rigid or isometric transformations, such as, articulations, bending, translation, and rotation. Here, we combine two such techniques, the continuous eccentricity transform [1-4, 12] and integral invariant signatures [5-6]. A detailed review of shape representation [7], matching and description techniques and categorical classification is given in [8].

The continuous eccentricity transform is used to find descriptors of shape based on geodesic distance maps. Such descriptors then yield histograms in the form of shape signatures. The signatures obtained by the eccentricity transform are considered to be invariant under rigid motion and isometric transformation of shape.

Compared to curvature measures [9], eccentricity transforms (Ecc) are robust to noise [1]. Ecc finds the geodesic distance for each point within a shape, to every other point on the boundary.

Faraz Janan is a DPhil Engineering Science candidate at the Institute of Bio-Medical Engineering (IBME), University of Oxford. Phone: +44 (0) 7550505969, e-mail: faraz.janan@some.ox.ac.uk.

Sir Michael Brady FRS FREng FMedSci, is Professor of Oncology at the Department of Oncology, University of Oxford UK.
It then assigns to each point a distance to the point farthest away from it. Instead of assigning the maximum distance, the mean distance may also be used. The geodesic distances for this purpose are calculated using the fast marching algorithm (FMA). The Ecc shape matching algorithm, matches histograms obtained from Ecc transformed images. Such a geodesic distance histogram does not explicitly contain boundary information, including information such as curvature, and it does not appear to have been used for establishing point-wise shape correspondences between shapes.

Conversely, integral invariants are shape descriptors that are preferred to curvature for their robustness to noise, and have been used effectively for shape matching [5] applications and for dividing shapes into further regions [6] to quantify occlusion and new growth. However, a fundamental problem with integral invariants is that they relate only to the boundary and do not take into account the information from inside the shape. As a result, two similar geometric features on a shape boundary, at very different geometrical locations will produce same shape signature. This may result in false matches in point-wise shape correspondence. We combine the two ideas, tuning integral invariant boundary signatures based on the eccentric information about locations within a shape. Figure 1 shows a shape with two pointed peaks, which have similar features, though in different locations. In the eccentricity transformed version of shape; it is immediately apparent that the two peaks now contain different values in the false colour model, and shown in Figure 2 using shape signatures.

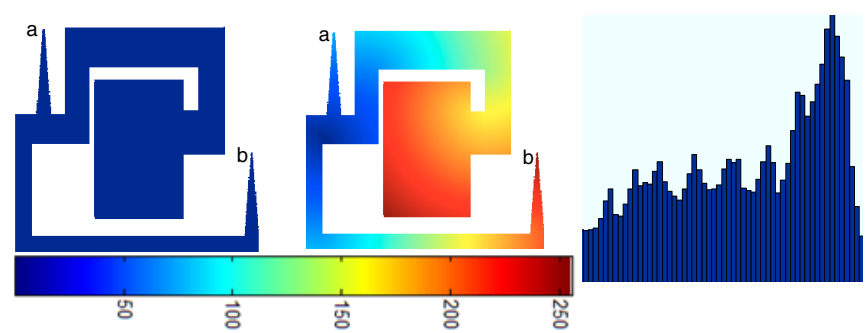

Figure 1: A shape (left), with its eccentricity transform (middle) and Ecc histogram shape signature (right)

In a typical shape correspondence application, this will help in establishing correct correspondences. Figure 3 shows two shapes, $S 1$ and $S 2$, with a pair of corresponding points, 
where $a 1$ corresponds to $a 2$ and $b 1$ corresponds to $b 2$. Both shapes are processed and corresponded as shown.

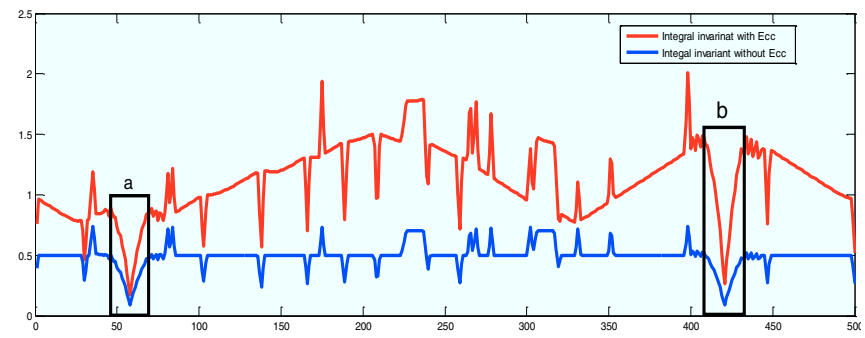

Figure 2: Normalized II signature (blue) of the original shape in Figure 1. The II invariant signature of the Ecc version (II-on-Ecc - red). The portions $a$ and $b$, show how two similar features may be tuned based on their locality using proposed method.
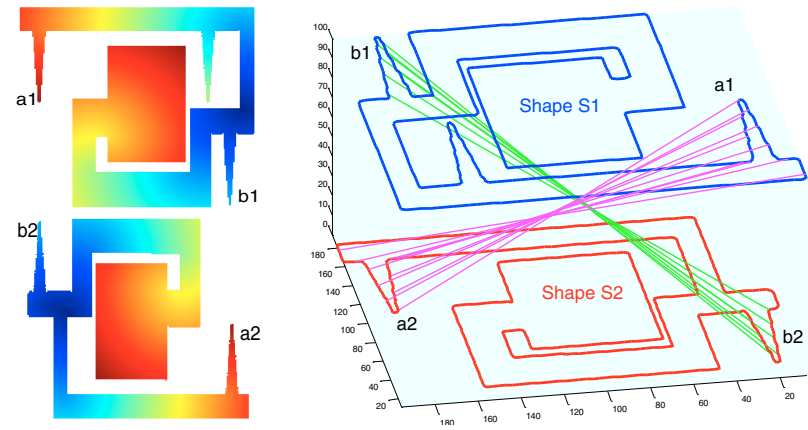

Figure 3: Two Ecc transformed shapes (left) and its correct correspondence (right) using II and fast marching algorithm. II without Ecc will incorrectly match points $b 1$ to $a 2$ and $a 1$ to $b 2$.

\section{ECCENTRICITY TRANSFORM}

Andrian [1] defines the eccentricity transform by considering a shape $S \subset \mathbb{R}^{2}$ with a smooth boundary $\partial S$, where $S$ may be an image $f_{s}$ of $n * m$ pixels, such that,

$$
f_{s}(x)=\left\{\begin{array}{l}
1 \text { for } x \in S \\
0 \text { otherwise }
\end{array}\right.
$$

The geodesic distance $d_{s}(x, y)$, between any two points $x$ and $y$ on the shape $\mathrm{S}$ is given by,

$d_{S}(x, y) \stackrel{\text { def }}{=} \underset{\gamma \in p(x, y)}{\min } L(\gamma)$, where $L(\gamma) \stackrel{\text { def }}{=} \int_{0}^{1}\left|\gamma^{\prime}(t)\right| d t$

Where, $p(x, y)$ is the set of paths $\gamma(t)$ from $x$ toy, such that

$$
\gamma(t)\left\{\begin{array}{l}
0 \text { for } t=x \\
1 \text { for } t=y
\end{array}\right.
$$

Inside the shape $S$ and for any starting point $x_{0}$, the distance function $U(x) \stackrel{\text { def }}{=} d\left(x_{0}, x\right)$ can be computed by finding solution of the Eikonal equation,

$$
\forall x \in S, \quad\|\nabla U(x)\|=1 \text {, and } U(0)=0
$$

The fast marching algorithm is used to solve the above Eikonal equation to find the minimum path between $x_{0}$ and $x$.
The eccentricity transform (Ecc) of $S$ to each point $p \in S$ is the shortest geodesic distance to the point on $S$, farthest away from it. In the feature set of the shape, where the distance for each point inside the shape is calculated to every point in the boundary, thus forming $I_{n} \times I_{m} \times n$ feature space, where $I_{n} \times I_{m}$ are the image dimensions and $n$ is the parameterization of the boundary curve $\partial S$.

$$
\operatorname{Ecc}_{S}(x) \stackrel{\text { def }}{=} \max _{y \in S} d_{S}(x, y)=\max _{y \in \partial S} d_{S}(x, y)
$$

The original paper $[1,12]$ on Ecc shape matching calculates a histogram to calculate the shape signature, without giving boundary correspondences. We have used integral invariants to perform shape matching and establish boundary correspondence.

\section{INTEGRAL INVARIANTS}

Hong defines (circular area) integral invariants in [5] by considering a disc $B_{r}(p)$ of radius $r$ applied to every point $p \in S$ of a closed contour $\partial S$, that is the boundary of the shape $S$. The characteristic function is then given by,

$$
\chi\left(B_{r}(p), \partial S\right)(x) \stackrel{\text { def }}{=}\left\{\begin{array}{c}
1 \text { if } x \in\left\{B_{r}(p) \cap \partial \dot{S}\right\} \\
0 \text { otherwise }
\end{array}\right.
$$

Where $\dot{\partial} S$ is the interior of the curve $\partial S$. The local integral area $I_{r}(\partial S)$ of the boundary curve $\partial S$ is given by the function $I_{r}(p)$ at every point $p$ with integral kernel $\chi$ as follows:

$$
I_{r}(p)=\int_{\Omega} \chi\left(B_{r}(p), \partial S\right)(x) d x
$$

Where $\Omega$ is the domain of the curve $\partial S$.

\section{INTEGRAL INVARIANTS ON ECCENTRICITY TRANSFORM SHAPES}

Once an Ecc image is acquired, a multi-scale approach is used for integral invariant (II) shape correspondence. $r$ is varied to span a range of apertures. As a result, integral invariant analysis creates a scale space where for every two points $x \in S 1$ and $y \in S 2$, the sum of squared difference of integral invariants is computed, and this forms a feature vector $V_{S}$. The largest singular value of $V_{S}$ is considered to be the maximum distance between $x$ and $y$. In this way a similarity/distance matrix $D\left(S_{1}, S_{2}\right)$ is obtained, which contains the integral invariant difference for each point between two shapes. For shape correspondence, the fast marching algorithm is applied to the similarity/distance matrix to find a distance map $\widehat{D}\left(S_{1}, S_{2}\right)$, and then the shortest geodesic path $G\left(S_{1}, S_{2}\right)$ from $D(0,0)$ to $D(n, n)$ is calculated using gradient descent algorithm, where $n$ is the parameterization of both boundary curves $\partial S_{1}$ and $\partial S_{2}$. An example of a similarity matrix is shown in Figure 7, with the 
geodesic map superimposed. Figure 4 shows the shape correspondence results of two man ('dude') shapes and its geodesic map path is overlaid on the distance map.
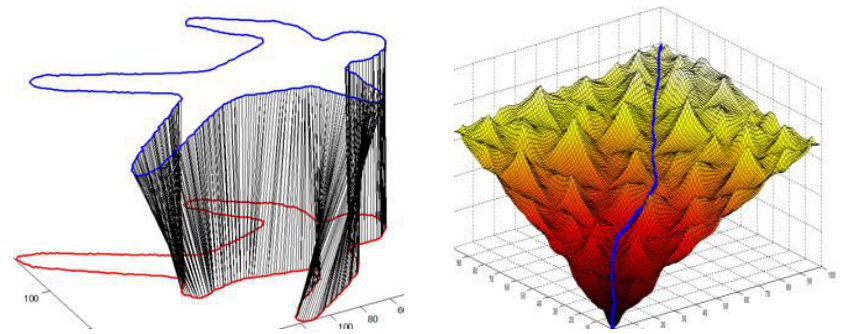

Figure 4: Left: point-wise correspondence of two human shapes, one with an occluded limb. Right: Distance map $\widehat{D}\left(S_{1}, S_{2}\right)$ computed by FMA, where Geodesic path $G\left(S_{1}, S_{2}\right)$ is calculated using gradient descent, shown as a blue line passing across the diagonal. The twist in the curve shows regularized correspondence of occluded limb.

The matching cost between two shapes is given by,

$$
C\left(S_{1}, S_{2}\right) \stackrel{\text { def }}{=} \int_{D\left(S_{1}, S_{2}\right)} G\left(S_{1}(t), S_{2}(t)\right) d t
$$

\section{RESULTS}

The algorithm has been applied to 36 shapes from Kimia database - 4 shapes each from 9 shape categories. As the method is general in nature, a broader evaluation may be carried out to evaluate precision of this method for a specific application. Though this algorithm is aimed to corresponding region of interest in temporal mammogram, we have used Kimia database as it is considered a standard to assess shape matching algorithms. All pairs of shapes are compared and a matching cost is calculated for II, Ecc and II-on-Ecc matching. II-on-Ecc gives the strongest intra group matching. Figure 5 \& Figure 9 summarize the result of matching.

Dark blocks along the diagonal reflect low cost of matching within a specific shape group, which means higher similarity.

The method is aimed to find changes in the region of interest, over time or in different views of the same mammogram. Figure 6 shows a pair of Craniocaudal (CC) and Mediolateral oblique (MLO) breast density maps created by Volpara [10]. Both mammograms were automatically segmented using a hierarchical segmentation method (topographic approach) based on iso-contours. As a result, a number of regions were segmented and were matched using the method described above. Two regions, suspected of abnormalities are shown in Figure 6. It may be noticed that II-on-Ecc performs better than II alone; the difference is clearly seen in Figure 7, where the geodesic path for II-on-Ecc shows a more regularised matching and consequently yield a lower matching cost for a closer match. Few more examples of this method applied to mammograms are given in Figure 8.

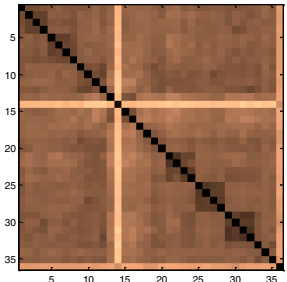

II

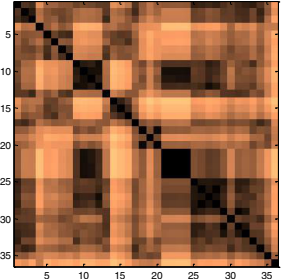

Ecc

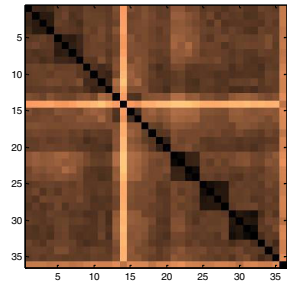

II-on-Ecc
Figure 5: Shape matching results of methods mentioned above. Dark pixels reflect a low matching cost and higher shape similarity, which is greater for II-on-Ecc. Refer to Figure 9 for shape retrieval details.
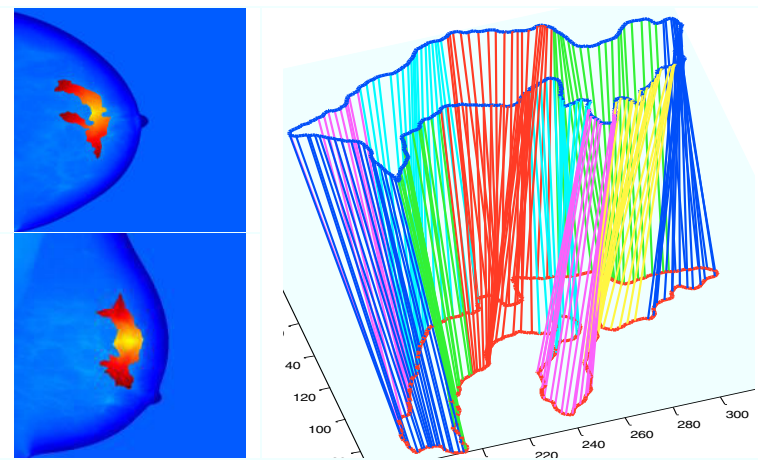

Figure 6: Segmented [11] pair of CC and MLO views of breast density maps, obtained by Volpara [10], and matched and corresponded using our proposed method.

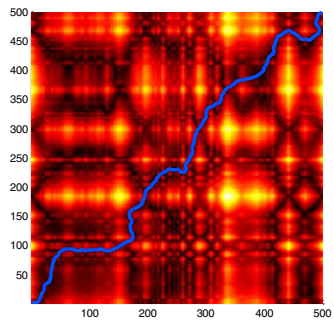

II

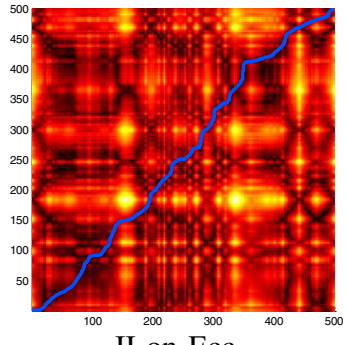

II-on-Ecc
Figure 7: Geodesic path drawn over similarity matrix, which shows point-wise correspondence between regions in Figure 6. It can be seen that more regularized correspondence is achieved by using II-on-Ecc, as compared to II alone. No results for Ecc given here, as it cannot establish point-wise correspondence of shapes.

\section{DISCUSSION}

In this paper, we have combined structural and boundary information in a shape matching application, applied to regional correspondence in temporal mammograms. Integral invariants and eccentricity transform are invariant to isometric deformations, such as bending and articulations. However, the integral invariants is a contour based local descriptor, which relates to the boundary of the shape and do not take into account inside the shape. On the other hand, Ecc is a global region based descriptor that maps the structural anatomy of a shape, however, does not explicitly contain the boundary information, including curvature. We describe a method that combines both techniques by tuning 
the integral invariant boundary signature based on the eccentric information about regions within the shape.

Experimental results here reflect upon the correct matching, which is a qualitative improvement compared to integral invariant results; however, the main aim of this method is to reduce the correspondence error while matching two shapes. Shape matching algorithms usually stuck in local minima while establishing point-wise correspondences. This method first stretches regional differences within each shape, thus elaborate dissimilarities before comparing them, which reduces the probability of false correspondence. This feature is the fundamental strength of our approach.

We have applied our method to shapes from various groups of Kimia database and have compared the results to those obtained by integral invariants and eccentricity transforms when applied separately. There is an overall improvement in results for both inter and intra group shape matching. The fast marching algorithm was applied to establish a point-wise correspondence between shapes and to calculate a matching cost. The results are encouraging and indicate scope for further improvement.

\section{REFERENCES}

[1] A. Ion, N. M. Artner, G. Peyré, W. G. Kropatsch, and L. D. Cohen, "Matching $2 D$ and $3 D$ articulated shapes using the eccentricity trans..," CVIU, vol. 115, pp. 817-834, 2011.

[2] A. Bildverarbeitung and D. Ion, "The Eccentricity Transform of n-Dimensional Shapes with and without Boundary," PhD Thesis, Vienna University of Tech., Vienna. 2009.

[3] A. Ion and G. Peyré, "Shape matching using the geodesic eccentricity transform - a study," 31st annual workshop of AAPR, Krumbach : Austria (2007).

[4] A. Ion, N. Artner, and G. Peyré, " $3 D$ shape matching by geodesic eccentricity," CVPRW, 2008.

[5] S. Manay, D. Cremers, B.-woo Hong, A. J. Y. Jr, and S. Soatto, "Integral Invariants for Shape Matching," PAMI, October, vol. 28, no. 10, pp. 1602-1618, 2006.

[6] F. Janan and S. Brady, "Region matching in the temporal study of mammograms using integral invariant scale-space," Breast Imaging, pp. 173-180, 2012.

[7] T. Sebastian, "On aligning curves", PAMI vol. 25, no. 1, pp. 116-124, 2003.

[8] A. Amanatiadis, V. G. Kaburlasos, a. Gasteratos, and S. E. Papadakis, "Evaluation of shape descriptors for shapebased image retrieval," IET Image Processing, vol. 5, no. 5, p. 493, 2011.

[9] S. Lie. Uber, "Differential invariants". Math. Ann., 1884

[10] R. Highnam, S. Brady, M. Yaffe, and N. Karssemeijer, "Robust Breast CompositionMeasurement-Volpara TM," Digital, 2010.

[11] B.-woo Hong and M. Brady, "Segmentation of mammograms in topographic approach," International Conference on Visual Information Engineering, pp. 157-160, 2003.

[12] www.numerical-tours.com

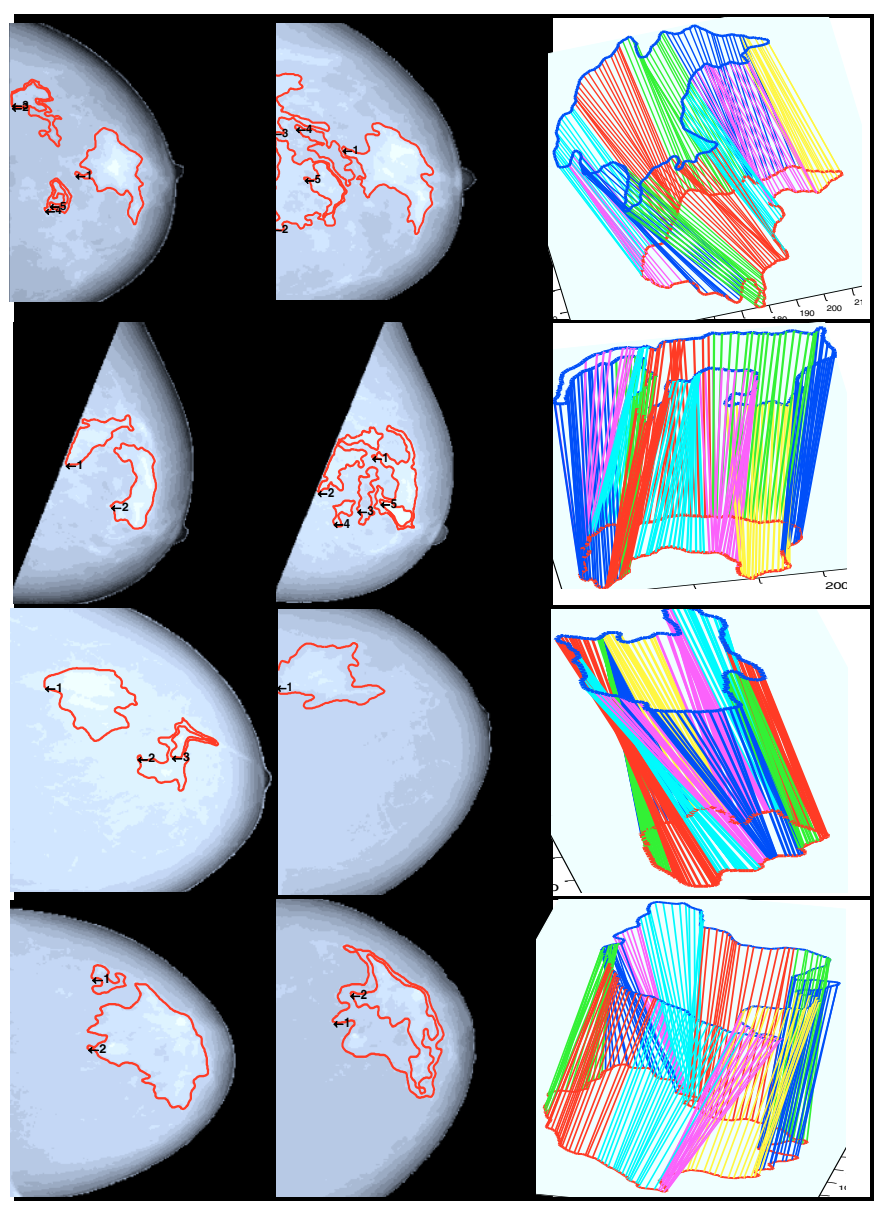

Figure 8: A few more examples of segmented, temporal mammograms, where regions are matched and corresponded against each other using the proposed method.

\begin{tabular}{|l|l|l|l|l|l|l|l|l|l||}
\hline & Rabbits & Alien & Fish & Hand & Jot & Tools & Man & Kite & Animals \\
\hline & & & & & & & & & \\
\hline
\end{tabular}

Figure 9: This chart shows the shape retrieval aspect of II-on-Ecc in Figure 5. This $\mathrm{X}$ and $\mathrm{Y}$ axis of the chart consists of shapes, which are indexed consecutively from 1-36 in 9 different shape groups from Kimia database. Each box represents a shape on $\mathrm{x}$ axis, and its height (range) on the y axis represent top 4 matches among all 36 shapes. The red bar in each box shows the median shape value of retrieved matches. Categories of Rabbits, Aliens, Tools, Men and Kite have perfect group retrieval results for II-onEcc method. 